\title{
CHRONIC TREATMENT WITH ETHANOL PRODUCES SUPERSENSITIVITY TO OXOTREMORINE
}

\author{
MARK J. MAJCHRZAK ${ }^{1}$ and STEVEN C. DILSAVER ${ }^{2}$ \\ ${ }^{1}$ Department of Psychiatry \\ University of Michigan, Ann Arbor, MI, U.S.A. \\ ${ }^{2}$ Psychopharmacology Program, Department of Psychiatry, \\ The Ohio state University, Columbus, OH, U.S.A.
}

(Final form, July 1989)

\section{Abstract}

Majchrzak, Mark J. and Steven C. Dilsaver: Chronic Treatment with Ethanol Produces Supersensitivity to oxotremorine. Prog. NeuroPsychopharmacol. \& Biol. Psychol., 1990, 14:379-386

1. The effect of chronic treatment with ethanol (148 $\mathrm{v}: \mathrm{v}$ in drinking water) on the physiological endpoint core temperature which is partially regulated by a muscarinic mechanism was measured in adult male rats $(n=8)$.

2. One and two weeks of treatment were associated with enhancement of the hypothermic response to oxotremorine, $0.25 \mathrm{mg} / \mathrm{kg}$ ip ( $\mathrm{p}=$ 0.0005 and $p=0.0001$, respectively).

3. The sample remained supersensitive to this muscarinic agonist 48 and 96 hours after the discontinuation of treatment ( $p=0.0014$ and $\mathrm{p}=0.013$ respectively).

4. Repeated injections of oxotremorine, $0.25 \mathrm{mg} / \mathrm{kg} \mathrm{ip}$, every other day for 10 days did not produce carry-over effects in a control experiment.

5. The results suggest that ethanol renders muscarinic mechanisms supersensitive during chronic treatment and that supersensitivity remains up to 96 hours following withdrawal.

Keywords: acetylcholine, alcohol, cholinergic, ethanol, muscarinic receptors, thermoregulation

Abbreviations: acetylcholine (Ach), intraperitoneally (ip), minutes (min), muscarinic acetylcholine receptor (mAchR), oxotremorine (OXO), $\left[{ }^{3} \mathrm{H}\right]$-quinuclidynl benzilate (QNB)

\section{Introduction}

Many non-psychedelic substances of abuse directly block the access of acetylcholine to the post-synaptic muscarinic acetylcholine receptor $(\mathrm{mAchR})$ or inhibit the release of this neurotransmitter from presynaptic neurons. Drugs with these properties include classical 
antimuscarinic agents, opiates, barbiturates, tetrahydrocannabinol, and ethanol (Dilsaver, 1986; Dilsaver, 1987). Ethanol decreases the release of Ach in various brain regions both in vivo and in vitro (Carmichael and Israel, 1975; Erikson and Graham, 1973; Hunt and Dalton, 1976). It might therefore partially denervate post-synaptic mAchRs thus supersensitizing muscarinic cholinergic networks. The authors tested this hypothesis by studying the capacity of orally administered ethanol to alter the hypothermic response to the muscarinic agonist oxotremorine.

\section{Material and Methods}

\section{Animals}

Adult, male Sprague-Dawley rats were purchased from Harlan taboratories (Indianapolis, IN, U.S.A.). The animals were housed in groups of 4 during treatment and were allowed food and water containing ethanol. The animals were placed in individual cages 16 hours prior to oxotremorine challenges.

A control experiment was conducted to demonstrate that multiple injections of oxotremorine $(0.15 \mathrm{mg} / \mathrm{kg})$ does not produce tolerance. Pharmaceutical Agents

oxotremorine (base) and methylscopolamine nitrate were purchased from Sigma Chemical Company (st. Louis, MO).

Drugs

Administration of Ethanol: Ethanol was administered by its addition to water ( 148 by volume). This was the sole drinking fluid.

oxotremorine challenge: Each oxotremorine challenge was conducted between 0930 and 1200 hours and was preceded by the administration of methylscopolamine nitrate, $1.0 \mathrm{mg} / \mathrm{kg}$ ip by 30 minutes, to block the peripheral effects of oxotremorine. Baseline temperature was measured 30 minutes later. This dose of methylscopolamine does not alter core temperature 30 minutes after its administration (Dilsaver, 1987). oxotremorine (base) $0.25 \mathrm{mg} / \mathrm{kg}$ ip, was administered following the baseline measurement of core temperature. Temperature was then recorded every 10 minutes for 120 minutes. 


\section{Temperature Measurement}

Thermosensors (Mini-Mitter Corp., Sun River, OR) were implanted into the peritoneal cavity of 8 adult, male sprague-Dawley rats weighing $206.9 \pm$ SEM. These devices emit radio waves at a rate proportional to core temperature. A transistor radio set to an $A M$ frequency served as a receiver. Time to emit 10 sounds was measured using a digital display stopwatch. This measure was converted to temperature using a linear regression equation, which was derived by measuring the emission rate of the thermosensors at three temperatures in a temperature-controlled water bath. This instrument allows the accurate detection of a change in temperature of $0.1^{\circ} \mathrm{C}$. Details regarding the calibration and use of the thermosensor are available elsewhere (Dilsaver et al, in press; Gallaher and Egner, 1987; ToccoBradley et al., 1985). Principles governing the use of measurements of core temperature in psychopharmacological research were the topic of a recent review (Dilsaver and Alessi, 1988).

\section{Statistical Analysis}

The thermic response to oxotremorine was measured at baseline, i.e., prior to the administration of ethanol, and at one-week intervals after that for two weeks. The thermic response to oxotremorine was also measured 48 and 96 hours after the discontinuation of treatment of ethanol. Significance of the hypothermic response to oxotremorine for a given challenge was determined by analysis of variance (ANOVA) with repeated measures. All measures of variance in the text refer to the standard error of the mean (SEM).

\section{Results}

\section{Experiment 1}

Mean core temperature prior to the first (baseline) challenge with oxotremorine was $38.2 \pm 0.3^{\circ} \mathrm{C}$. The mean core temperature for the $120-$ min period following the first challenge with oxotremorine was 38.1 $\pm 0.2^{\circ} \mathrm{C}$ (ns). Treatment with ethanol for 7 days produced a mean hypothermic response of $0.47 \pm 0.11^{\circ} \mathrm{C}$ and a highly significant enhancement of the hypothermic response as a function of time relative to baseline $(p=0.0005, f=3.36, d f=12 / 84)$. Fourteen days of treatment with ethanol resulted in further enhancement of the hypothermic response to $0.59 \pm 0.09^{\circ} \mathrm{C}$. Further, the change in mean 
hypothermic response as a function of time was highly significant (p $<0.0001, f=4.45$, df $=12 / 84$ ) (Table 1 ).

The mean hypothermic response after 48 hours of abstinence was 0.47 $\pm 0.11^{\circ} \mathrm{C}$. The hypothermic response as a function of time relative to baseline was significant $(p=0.0014, f=3.03, \mathrm{df}=12 / 84)$. The hypothermic response after 96 hours of abstinence was $0.54 \pm 0.7^{\circ} \mathrm{C}$ and the thermic response as a function of time remained enhanced ( $p=$ $0.013, f=2.31, d f=12 / 84$ ) (Fig 1).

The average weight of the animals during the period of ethanol administration increased by an average of $29 \pm 5.2 \mathrm{~g}$ per week. The animals remained healthy throughout the study.

Table 1

Summary of Findings and Statistical Analysis

\section{Mean Core Temperature:}

Before treatment with ethanol

$38.2 \pm 0.3^{\circ} \mathrm{C}$

Hypothermic Response

After first challenge with oxotremorine:

Following 7 days of treatment with ethanol:

Following 14 days of treatment with ethanol:

$-0.1 \pm 0.1^{\circ} \mathrm{C}$

$-0.47 \pm 0.11^{\circ} \mathrm{C}$

$-0.59 \pm 0.11^{\circ} \mathrm{C}$

Significance of hypothermic response as a function of time:

(two-way ANOVA for repeated measures)

After first challenge with oxotremorine

Following 7 days of treatment

Following 14 days of treatment

not significant

$\mathrm{p}=0.0005, \mathrm{~F}=3.36, \mathrm{df}=12 / 84$

$\mathrm{p}<0.0001, \quad \mathrm{~F}=4.45, \mathrm{df}=12 / 84$

\section{Experiment 2 (Control Experiment).}

The hypothesis subjected to testing in the main experiment is that "chronic treatment with ethanol will render animals supersensitive to the hypothermic effects of a muscarinic agonist." The experimental design required multiple injections of oxotremorine, $0.25 \mathrm{mg} / \mathrm{kg}$ ip. This raises the possibility of carry-over effects. Repeated injections of cholinomimetic agents could produce subsensitivity to subsequent challenges (Dilsaver, 1986; Marks and Collins, 1985; overstreet, et al, 1973). We challenged animals ( $n=8$, mean weight $\pm \mathrm{SEM}=304.7 \pm 5.2$ ) with oxotremorine, $0.25 \mathrm{mg} / \mathrm{kg} \mathrm{ip}$, every other day for 10 days. Each of the 5 challenges was preceded by the injection of methylscopolamine nitrate, $1.0 \mathrm{mg} / \mathrm{kg} \mathrm{ip}$, by 30 minutes. The mean hypothermic response on Day 1 was $0.28 \pm 0.11^{\circ} \mathrm{C}$. The mean response as 
a function of time did not differ across time, i.e., between challenges $1(p=0.50, F=0.96$, df $=12 / 84), 3(p=0.34, f=1.13$, $d f=12 / 84)$, and $5(p=0.66, f=0.79, d f=12 / 84)$. Thus, there was no detectible change in the thermic responsiveness to oxotremorine.

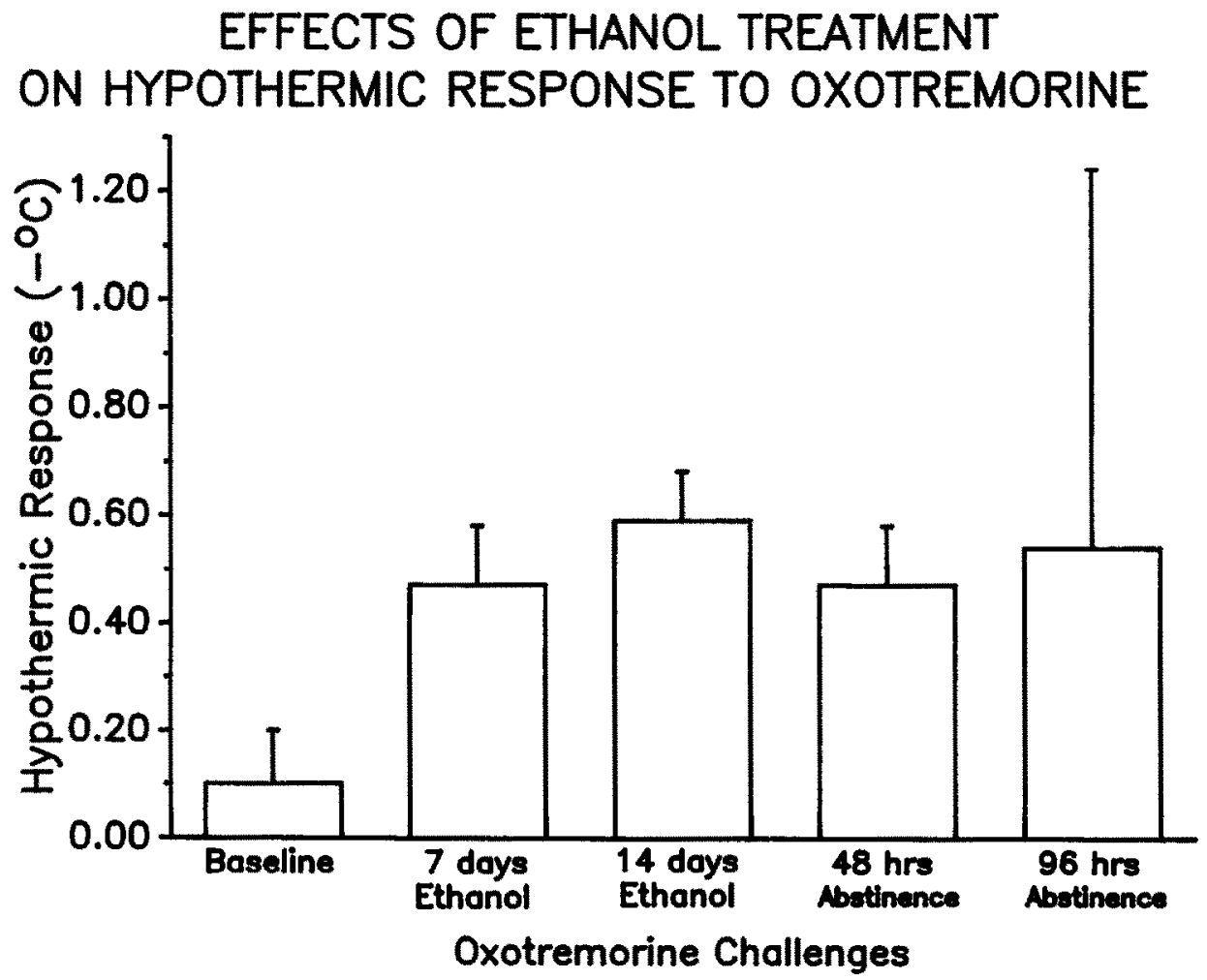

Fig 1. This illustrates the ethanol-induced increase in the mean hypothermic response of the experimental group $(n=8)$ to the muscarinic cholinergic agonist OXO $(0.25 \mathrm{mg} / \mathrm{kg}$ ip) relative to baseline after 7 and 14 days of treatment ( $14 \%$ by volume) and after 48 and 96 hours of abstinence. Methylscopolamine nitrate (1 mg/ $\mathrm{kg}$ ip) was administered $30 \mathrm{~min}$ before oxo. Core temperature was measured every $10 \mathrm{~min}$ for 120 min following the injection of oxo. The mean hypothermic response of a given rat is the average of the 12 deviations from the animal's core temperature at $t=0$. The mean hypothermic response of the group to oxo was significantly elevated at all four points depicted in this illustration. Please see the results section for the results of an analysis of hypothermic response as a function of time. 


\section{Discussion}

\section{Interpretation}

Drugs which inhibit the release of Ach indirectly denervate postsynaptic mAchRs and should therefore affect mAchR receptor upregulation and/or the supersensitization of cholinoreceptive neurons. The data we present support this. Our data are consistent with electrophysiological findings that ethanol enhances the excitatory response of hippocampal neurons to iontophoretically applied Ach (Mancillas et al, 1986).

\section{Supporting Evidence}

Treatment with ethanol upregulates mAchRs in rodents. Tabakoff et al (1979) observed that seven days of treatment with ethanol produced a $115 \%$ to $125 \%$ increase in the density of mAchRs radioligand binding sites in mouse brain. Smith (1973) gave ethanol, $7 \%$ by volume, to mice for 8 days and observed that this treatment produced severe dependence as evidenced by whole body tremor and convulsions upon routine handling. A cholinergically-enriched synaptosomal fraction from the brains of these animals showed a $28 \%$ increase in specific (QNB) binding. Rabin et al (1980) also found that this dose of ethanol increased the density of QNB binding sites in the hippocampus and cerebral cortex of mice. Very long term (75 weeks) treatment with ethanol (10\%) produced a significant increase in mAchR radioligand binding sites which was detected 8 days following withdrawal (Nordberg and Wahlstrom, 1982). Upregulation of mAchRs persists for up to 4 weeks following the withdrawal of ethanol.

The enhanced responsiveness to oxotremorine observed in this study suggests that chronic ethanol treatment supersensitizes cholinoceptive neurons. Supersensitization of the muscarinic system is evident up to 96 hours following withdrawal of ethanol. The study was not designed to ascertain the decay of this phenomenon. This requires another study. Documentation that repeated challenges with oxotremorine, $0.25 \mathrm{mg} / \mathrm{kg}$, did not produce carry-over effects supports the conclusion that chronic treatment with ethanol results in the supersensitization of cholinergic mechanisms. 
Conclusions

Several commonly abused drugs inhibit the release of acetylcholine. These drugs include ethanol, barbiturates, opiates, and delta ${ }^{9-}$ tetrahydrocannabinol. Pharmacological agents potently inhibiting the release of acetylcholine at muscarinic cholinergic junctions are expected to produce denervation supersensitivity of the post-synaptic neuron to acetylcholine and muscarinic agonists. This would likely be accompanied by the upregulation of post-synaptic muscarinic receptors. Investigators previously demonstrated that treatment with ethanol affects an increase in the density of treated quiniclidinylbenzilate $\left(\left[{ }^{3} \mathrm{H}\right] \mathrm{QNB}\right)$ binding sites. $Q N B$ is a ligand binding to muscarinic acetylcholine receptors. This study tested the hypothesis that chronic treatment with ethanol produces supersensitivity to muscarinic agonists. Seven days of treatment with ethanol (14\% v:v in drinking water) was sufficient to enhance the thermic response to oxotremorine $(0.25 \mathrm{mg} / \mathrm{kg} \mathrm{ip})$. The thermic response to oxotremorine remained elevated 96 hours following the conclusion of two weeks of treatment with ethanol.

\section{Acknowledgements}

Supported in part by Physician Scientist Career Development Award, Grant \#SRC1K11 MH00553-02, (Muscarinic Receptor Abnormalities in Affective Illness) awarded by the National institute of Mental Health.

\section{References}

CARMICHAEL, F. J. and ISRAEL Y. (1975) Effects of ethanol on neurotransmitter release by rat brain cortical slices. J. Pharmacol. Expt. Therap. 193: 824-834.

DILSAVER, S. C. (1987) The pathophysiologies of substance abuse and affective disorders: An integrative model? J. Clin. Psychopharmacol. 7: 1-10.

DILSAVER, S. C. (1986) Pharmacologic induction of cholinergic system upregulation and supersensitivity in affective disorders research. J. Clin. Psychopharmacol. $\underline{6}$ : 65-74.

DILSAVER, S.C., and ALESSI, N.E. (1988) Temperature as a dependent variable in the study of cholinergic mechanisms. Prog. NeuroPsychopharma. Biol. Psych. 12: 1-32.

DILSAVER, S.C., MAJCHRZAK, M.J., and ALESSI, N.E. (in press) Telemetric measurement of core temperature in psychobiological research: Reliability and validation. Prog. Neuro-Psychopharma. Biol. Psych.

DILSAVER, S.C., SNIDER. R. M. , and ALESSI, N. E. (1987) Amitriptyline supersensitizes a central cholinergic mechanism. Biol. Psychiatry. 22: $495-507$. 
ERIKSON, C. K. and GRAHAM, D. T. (1973) Alternation of cortical and reticular acetylcholine release by ethanol in viva. J. Pharmacol. Exp. Ther. 185: 583-593.

GALLAHER, E. J. and EGNER, D..A. (1987) Rebound hyperthermia follows ethanol- induced hypothermia in rats. Psychopharmacology 91: 3439.

HUNT, W. A. and DALTON, T. K. (1976) Regional brain acetylcholine levels in rats acutely treated with ethanol or rendered ethanoldependent. Brain Research 109: 628-631.

MANCILLAS, J. R., SIGGINS, G.R. and BLOOM, F. E. (1986) Systemic ethanol: Selective enhancement of responses to acetylcholine and somatostatin in hippocampus. Science 231: 161-163.

MARKS, J. J. and COLLINS, A. C. (1985) Tolerance, cross-tolerance, and receptors after chronic nicotine and oxotremorine. Pharmacol. Biochem. and Behavior. 22: 283-291.

NORDBERG, A. and WAHLSTROM, G. (1982) Tolerance, physical dependence and changes in muscarinic receptor binding sites after chronic ethanol treatment in the rat. Life sciences 31: 277-287.

OVERSTREET, D. H., KOZAR, M. P. and LYNCH, G. S. (1973) Reduced hypothermic effects of cholinomimetic agents following chronic anticholinesterase treatment. Neuropharmacology 12: 1017-1032.

RABIN, R. A., WOLFE, B. B., DIBNER, M. D., AZHNISER, N.R., MELCHIOR, C. and MOLINOFF, P. B. (1980) Effects of ethanol administration and withdrawal on neurotransmitter receptor systems in c57 mice. J. Pharmacol. Expt. Therap. 213:491-496.

SMITH, T. L. (1973) Influence of chronic ethanol consumption on muscarinic cholinergic receptors and their linkage to phospholipid metabolism in mouse synaptosomes. Neuropharmacology $\underline{22}$ : 661-663.

TABAKOFF, B., MUNOZ-MARCUS, M., FIELDS, J. Z. (1979) Chronic ethanol feeding produces an increase in muscarinic cholinergic receptors in mouse brain. Life. Sci. 25: 2173-2180.

TOCCO-BRADLEY, R., KLUGER, M. J. and KAUFFMAN, C. A. (1985) Effect of age on fever: An acute phase response of rats to endotoxin and salmonella Typhimurium. Infect. Immun. 47: 106-111.

Inquiries and reprint requests should be addressed to:

Steven C. Dilsaver, M.D.

Department of Psychiatry

The ohio State University

473 West 12th Avenue

Columbus, Ohio 43210-1228 